PROCEEDINGS OF THE

AMERICAN MATHEMATICAL SOCIETY

Volume 129, Number 10, Pages 3009-3015

S 0002-9939(01)06041-5

Article electronically published on March 15, 2001

\title{
SOME RESULTS RELATED TO THE CORACH-PORTA-RECHT INEQUALITY
}

\author{
AMEUR SEDDIK
}

(Communicated by Joseph A. Ball)

\begin{abstract}
Let $L(H)$ be the algebra of all bounded operators on a complex Hilbert space $H$ and let $S$ be an invertible self-adjoint (or skew-symmetric) operator of $L(H)$. Corach-Porta-Recht proved that
\end{abstract}

$(*)$

$\forall X \in L(H),\left\|S X S^{-1}+S^{-1} X S\right\| \geq 2\|X\|$.

The problem considered here is that of finding (i) some consequences of the Corach-Porta-Recht Inequality; (ii) a necessary condition (resp. necessary and sufficient condition, when $\sigma(P)=\sigma(Q))$ for the invertible positive operators $P, Q$ to satisfy the operator-norm inequality $\left\|P X P^{-1}+Q^{-1} X Q\right\| \geq 2\|X\|$, for all $X$ in $L(H)$; (iii) a necessary and sufficient condition for the invertible operator $S$ in $L(H)$ to satisfy $(*)$.

\section{INTRODUCTION}

All operators considered here are bounded operators on a complex Hilbert space $H$. The collection of operators in $H$ is denoted by $L(H)$.

For $T \in L(H)$, we denote by $\sigma(T), \operatorname{co}(\sigma(T)), r(T), W_{0}(T),\{T\}^{\prime}$ and $\{T\}^{\prime \prime}$ the spectrum, the convex hull of the spectrum, the spectral radius, the numerical range, the commutant and the bicommutant of $T$, respectively.

If $A=\left(a_{i j}\right)$ and $B=\left(b_{i j}\right)$ are two complex $n \times n$ matrices, then define the Schur (or Schur-Hadamard) product of $A$ and $B$ to be the matrix $A \circ B=\left(a_{i j} b_{i j}\right)$.

In [1, Corach, Porta, and Recht have proved that for any invertible self-adjoint or skew-symmetric operator $S$, the operator-norm inequality

$$
\left\|S X S^{-1}+S^{-1} X S\right\| \geq 2\|X\|
$$

holds for all operators $X$.

It is also clear that, for any invertible operator $S$ and for any two invertible positive operators $P, Q$, we have

(a) $0<\inf _{\|X\|=1}\left\|P X P^{-1}+Q^{-1} X Q\right\| \leq 2$,

(b) $0 \leq \inf _{\|X\|=1}\left\|S X S^{-1}+S^{-1} X S\right\| \leq 2$.

It may be seen by the Corach-Porta-Recht Inequality that the infimum in (a) is 2 , if $P=Q$; and the infimum in (b) is also 2 , for $S$ an invertible self-adjoint operator,

Received by the editors February 29, 2000.

2000 Mathematics Subject Classification. Primary 47A30, 47B15.

Key words and phrases. Operator-norm inequality, self-adjoint operator, positive operator.

(C)2001 American Mathematical Society 
or more generally, if $S$ is of the form $S=\lambda M$, where $M$ is an invertible self-adjoint operator and $\lambda$ is a nonzero scalar.

The purpose of this paper is the following:

(1) In $\S 2$, we give the following consequences of the Corach-Porta-Recht Inequality. For all invertible positive commuting operators $P, Q$ and for all operators $X$, we have

(i) $\left\|P X P^{-1}+Q^{-1} X Q\right\| \geq 2\|X\|$, if $\|X\|=r(X)$,

(ii) $\max \left\{\left\|P X P^{-1}+Q^{-1} X Q\right\|,\left\|P X^{*} P^{-1}+Q^{-1} X^{*} Q\right\|\right\} \geq 2\|X\|$,

(iii) $\left\|n X+P X P^{-1}+P^{-1} X P\right\| \geq(n+2)\|X\|$, for $n=0,1,2$.

(2) In $\S 3$, we show that the infimum in (a) is 2 only if $\{P\}^{\prime}=\{Q\}^{\prime}$; on the other hand, if $\sigma(P)=\sigma(Q)$, then the infimum in (a) is 2 if and only if $P=Q$.

(3) In $\S 4$, we show that the only operators $S$ for which the infimum in (b) is 2 are those of the form $S=\lambda M$, where $M$ is an invertible self-adjoint operator and $\lambda$ is a nonzero scalar.

\section{Some Consequences of the Corach-Porta-Recht Inequality}

Lemma 2.1 ([1]). For an invertible self-adjoint or skew-symmetric operator $S$, we have $\forall X \in L(H):\left\|S X S^{-1}+S^{-1} X S\right\| \geq 2\|X\|$.

Theorem 2.2. For any pair $(P, Q)$ of commuting invertible positive operators and for any $X \in L(H)$ such that $\|X\|=r(A)$, we have

$$
\left\|P X P^{-1}+Q^{-1} X Q\right\| \geq 2\|X\| .
$$

Proof. Let $X \in L(H)$ such that $\|X\|=r(A)$ and put $Y=P^{\frac{1}{2}} Q^{-\frac{1}{2}} X Q^{\frac{1}{2}} P^{-\frac{1}{2}}$. Then since $P^{\frac{1}{2}} Q^{\frac{1}{2}}=Q^{\frac{1}{2}} P^{\frac{1}{2}}$ is self-adjoint, we have by Lemma 2.1

$$
\begin{aligned}
\left\|P X P^{-1}+Q^{-1} X Q\right\| & =\left\|\left(P^{\frac{1}{2}} Q^{\frac{1}{2}}\right) Y\left(P^{\frac{1}{2}} Q^{\frac{1}{2}}\right)^{-1}+\left(P^{\frac{1}{2}} Q^{\frac{1}{2}}\right)^{-1} Y\left(P^{\frac{1}{2}} Q^{\frac{1}{2}}\right)\right\| \\
& \geq 2\|Y\| \\
& \geq 2 r(X) \\
& \geq 2\|X\| .
\end{aligned}
$$

Theorem 2.3. For any pair $(P, Q)$ satisfying the condition of Theorem 2.2 and for any operator $X$, we have

$$
\max \left\{\left\|P X P^{-1}+Q^{-1} X Q\right\|,\left\|P X^{*} P^{-1}+Q^{-1} X^{*} Q\right\|\right\} \geq 2\|X\| .
$$

Proof. For $X \in L(H)$, let

$$
A=\left[\begin{array}{ll}
P & 0 \\
0 & P
\end{array}\right], B=\left[\begin{array}{ll}
Q & 0 \\
0 & Q
\end{array}\right] \text { and } Y=\left[\begin{array}{ll}
0 & X \\
X^{*} & 0
\end{array}\right] .
$$

The pair $(A, B)$ satisfies the condition of Theorem 2.2 and $\|Y\|=r(Y)$ (since $Y$ is self-adjoint). Then we have

$$
\begin{aligned}
\left\|A X A^{-1}+B^{-1} X B\right\| & =\| \begin{array}{cc}
0 & P X P^{-1}+Q^{-1} X Q \\
P X^{*} P^{-1}+Q^{-1} X^{*} Q & 0
\end{array} \\
& \geq 2\|Y\|=2\|X\| l,
\end{aligned}
$$

i.e.

$$
\max \left\{\left\|P X P^{-1}+Q^{-1} X Q\right\|,\left\|P X^{*} P^{-1}+Q^{-1} X^{*} Q\right\|\right\} \geq 2\|X\| .
$$


Theorem 2.4. For any invertible positive operator $P$, and for $n=0,1,2$, we have

$$
\forall X \in L(H):\left\|n X+P X P^{-1}+P^{-1} X P\right\| \geq(n+2)\|X\| .
$$

Proof. If $n=0,(1)$ follows from Lemma 2.1 .

For all $X$, we have

$$
\begin{aligned}
\left\|2 X+P X P^{-1}+P^{-1} X P\right\|= & \| P^{\frac{1}{2}}\left(P^{\frac{1}{2}} X P^{-\frac{1}{2}}+P^{-\frac{1}{2}} X P^{\frac{1}{2}}\right) P^{-\frac{1}{2}} \\
& +P^{-\frac{1}{2}}\left(P^{\frac{1}{2}} X P^{-\frac{1}{2}}+P^{-\frac{1}{2}} X P^{\frac{1}{2}}\right) P^{\frac{1}{2}} \| \\
\geq & 2\left\|P^{\frac{1}{2}} X P^{-\frac{1}{2}}+P^{-\frac{1}{2}} X P^{\frac{1}{2}}\right\| \\
\geq & 4\|X\|,
\end{aligned}
$$

that is, (1) is true for $n=2$.

It follows from the case $n=2$, that for all $X$, we have

$$
\begin{aligned}
\left\|X+P X P^{-1}+P^{-1} X P\right\| & \geq\left\|2 X+P X P^{-1}+P^{-1} X P\right\|-\|X\| \\
& \geq 3\|X\| .
\end{aligned}
$$

Remark 2.1. In the cases $n=1$ and $n=2$, the relation (1) is false in general if we replace the condition "positive" by the condition "self-adjoint"; this may be seen by the following example:

$$
P=\left[\begin{array}{ll}
1 & 0 \\
0 & -1
\end{array}\right] \text { and } X=\left[\begin{array}{ll}
0 & 1 \\
1 & 0
\end{array}\right]
$$

Then

$$
\left\{\begin{array}{l}
\left\|X+P X P^{-1}+P^{-1} X P\right\|=1<3=3\|X\| \\
\left\|2 X+P X P^{-1}+P^{-1} X P\right\|=0<4=4\|X\| .
\end{array}\right.
$$

\section{OPERATOR-NORM INEQUALITY AND POSITIVE OPERATORS}

Definition 3.1. An operator $A$ in $L(H)$ is called convexoid if $W_{0}(A)=\operatorname{co\sigma }(A)$.

Lemma 3.1 ([2]). Let $A \in L(H)$. If $\|A-\alpha\|=r(A-\alpha)$, for all complex $\alpha$, then A is convexoid.

Lemma 3.2. Let $P$ and $Q$ be in $L(H)$ such that $P>0$ and $Q>0$. If we have

$$
\forall X \in L(H):\|X\|+\left\|P X P^{-1}\right\| \geq 2\left\|Q X Q^{-1}\right\|
$$

then $\{P\}^{\prime} \subset\{Q\}^{\prime}$.

Proof. (i) Let $X$ be self-adjoint such that $P X=X P$, and let $\alpha$ be a complex number. Then, by $(2),\|X-\alpha\| \geq\left\|Q(X-\alpha) Q^{-1}\right\|$, and since $X-\alpha$ is normal, we also have $\left\|Q(X-\alpha) Q^{-1}\right\| \geq\|X-\alpha\|$, so that $\left\|Q(X-\alpha) Q^{-1}\right\|=\|X-\alpha\|$. Then, by Lemma 3.1, we have $W_{0}\left(Q X Q^{-1}\right)=\operatorname{co\sigma }(X)$, and since $X$ is self-adjoint, we obtain $Q X Q^{-1}=Q^{-1} X Q$, and also $Q X=X Q$.

(ii) Now let $X=X_{1}+i X_{2}$, where $X_{1}=\operatorname{Re} X$ and $X_{2}=\operatorname{Im} X$, such that $P X=X P$. Then, we have $P X_{1}=X_{1} P$ and $P X_{2}=X_{2} P$; from (i) it follows that $Q X_{1}=X_{1} Q$ and $Q X_{2}=X_{2} Q$, and also $Q X=X Q$; we conclude that $\{P\}^{\prime} \subset$ $\{Q\}^{\prime}$. 
Theorem 3.3. Let $P$ and $Q$ be in $L(H)$ such that $P>0$ and $Q>0$. If we have

$$
\forall X \in L(H):\left\|P X P^{-1}\right\|+\left\|Q^{-1} X Q\right\| \geq 2\|X\|,
$$

then $\{P\}^{\prime}=\{Q\}^{\prime}$.

Proof. From (3), we have

$$
\forall X \in L(H):\|X\|+\left\|P Q X Q^{-1} P^{-1}\right\| \geq 2\left\|Q X Q^{-1}\right\| .
$$

Let $U M$ be the polar decomposition of $P Q\left(U\right.$ is unitary and $\left.M=\left(Q P^{2} Q\right)^{\frac{1}{2}}\right)$. Then, from (4), we obtain

$$
\forall X \in L(H):\|X\|+\left\|M X M^{-1}\right\| \geq 2\left\|Q X Q^{-1}\right\|
$$

and, by Lemma 3.2, we have $M Q=Q M$; then $P Q=Q P$.

Now let $X$ be self-adjoint such that $P X=X P$ and let $\alpha$ be a complex number. Therefore, $Q X Q^{-1} \in\{P\}^{\prime}$ and, from (3), we obtain

$$
\forall X \in L(H):\left\|Q(X-\alpha) Q^{-1}\right\| \leq\|X\| \text {. }
$$

It follows that $Q X=X Q$, so that $\{P\}^{\prime} \subset\{Q\}^{\prime}$.

The symmetric roles of $P, Q$ in (3) also give $\{Q\}^{\prime} \subset\{P\}^{\prime}$, and finally we have $\{P\}^{\prime}=\{Q\}^{\prime}$.

Corollary 3.4. Let $P$ and $Q$ be in $\mathcal{L}(H)$ such that $P>0$ and $Q>0$. If we have

$$
\forall X \in \mathcal{L}(H):\left\|P X P^{-1}+Q^{-1} X Q\right\| \geq 2\|X\|,
$$

then $\{P\}^{\prime}=\{Q\}^{\prime}$.

Proof. Since we have $\left\|P X P^{-1}\right\|+\left\|Q^{-1} X Q\right\| \geq\left\|P X P^{-1}+Q^{-1} X Q\right\|$, for all operators $X$, the result follows immediately by Theorem 3.3.

Lemma 3.5. Let $\varepsilon>0$ and let $\alpha_{1}, \ldots, \alpha_{n}, \beta_{1}, \ldots, \beta_{n}$ (for $n \in \mathbb{N}^{*}$ ) such that $0<$ $\alpha_{1}<\cdots<\alpha_{n} \leq 1,\left\{\alpha_{1}, \ldots, \alpha_{n}\right\}=\left\{\beta_{1}, \ldots, \beta_{n}\right\}$ and $\frac{\alpha_{i}}{\alpha_{j}}+\frac{\beta_{j}}{\beta_{i}} \geq 2-\varepsilon$, for all $i, j$. Then we have $\left|\alpha_{i}-\beta_{i}\right|<\varepsilon$, for all $i$.

Proof. From the hypothesis, we obtain $\beta_{i}-\beta_{j}<\varepsilon$, if $i<j$.

Let $i \in\{1, \ldots, n\}$ such that $\alpha_{i} \neq \beta_{i}$ (in the case $\alpha_{i}=\beta_{i}$, of course we have $\left.\left|\alpha_{i}-\beta_{i}\right|=0<\varepsilon\right)$.

There are three cases $i=1, i=n$ and $1<i<n$.

Case 1. $i=1$. There exists $j \geq 2$, such that $\beta_{j}=\alpha_{1}$, so we have $\left|\beta_{1}-\alpha_{1}\right|=$ $\beta_{1}-\beta_{j}<\varepsilon$, since $j>1$.

Case 2. $i=n$. There exists $j<n$, such that $\beta_{j}=\alpha_{n}$, so we have $\left|\beta_{n}-\alpha_{n}\right|=$ $\beta_{j}-\beta_{n}<\varepsilon$, since $n>j$.

Case 3. $1<i<n$. If $\beta_{i}>\alpha_{i}$, then there exists $j>i$, such that $\beta_{j} \leq \alpha_{i}$, and we have $\left|\beta_{i}-\alpha_{i}\right|=\beta_{i}-\beta_{j}<\varepsilon$, since $j>i$. If $\beta_{i}<\alpha_{i}$, then there exists $j<i$, such that $\left|\beta_{i}-\alpha_{i}\right|=\beta_{j}-\beta_{i}<\varepsilon$, since $i>j$.

Theorem 3.6. Let $P$ and $Q$ be in $L(H)$ such that $P>0, Q>0$ and $\sigma(P)=\sigma(Q)$. Then the following properties are equivalent:

(i) $\forall X \in L(H),\left\|P X P^{-1}\right\|+\left\|Q^{-1} X Q\right\| \geq 2\|X\|$.

(ii) $P=Q$. 
Proof. We may assume, without loss of the generality, that $\|P\|=\|Q\|=1$.

(i) implies (ii). Decompose $P$ and $Q$ using the spectral measure

$$
P=\int \lambda d E_{\lambda}, \quad Q=\int \lambda d F_{\lambda}
$$

and consider

$$
P_{n}=\int h_{n}(\lambda) d E_{\lambda}=h_{n}(P), \quad Q_{n}=\int h_{n}(\lambda) d F_{\lambda}=h_{n}(Q)
$$

where $h_{n}(\lambda)$ is a function of the form

$$
h_{n}(\lambda)=\frac{k}{n}, \text { for } \frac{k}{n} \leq \lambda<\frac{k+1}{n} \text {, and } k=0,1,2, \ldots .
$$

Then by the spectral theorem and by the form of $h_{n}(\lambda)$, we have $\sigma\left(P_{n}\right)=$ $\sigma\left(Q_{n}\right)=h_{n}(\sigma(P))$ is finite, $P_{n} \longrightarrow P, Q_{n} \longrightarrow Q$ (uniformly) and $P_{n} \in\{P\}^{\prime \prime}, Q_{n} \in$ $\{Q\}^{\prime \prime}$ (where $\{P\}^{\prime \prime}=\{Q\}^{\prime \prime}$, by Theorem 3.3).

Put $\sigma\left(P_{n}\right)=\left\{\alpha_{1}, \ldots, \alpha_{p}\right\}$ such that $0<\alpha_{1}<\cdots<\alpha_{p} \leq 1$. Then there exist $p$ orthogonal projections $E_{1}, \ldots, E_{p}$ such that $E_{i} E_{j}=E_{j} E_{i}=0$ if $i \neq j$, $E_{1} \oplus \ldots \oplus E_{p}=I$ and $P_{n}=\sum_{i=1}^{p} \alpha_{i} E_{i}$.

Since $\sigma\left(P_{n}\right)=\sigma\left(Q_{n}\right), P_{n} Q_{n}=Q_{n} P_{n}$ and $Q_{n}$ is normal, there exist $p$ scalar $\beta_{1}, \ldots, \beta_{p}$ such that $Q_{n}=\sum_{i=1}^{p} \beta_{i} E_{i}$ and $\left\{\alpha_{1}, \ldots, \alpha_{p}\right\}=\left\{\beta_{1}, \ldots, \beta_{p}\right\}$.

Let $\varepsilon>0$. Then there exists an integer $N$ such that

$$
\forall n>N, \forall X \in L(H),\left\|P X P^{-1}\right\|+\left\|Q^{-1} X Q\right\| \geq(2-\varepsilon)\|X\| .
$$

Let $n>N$ and $X_{i j}=E_{i} X E_{j}$, for $X \in L(H)$. Then, by using (*), we have

$$
\frac{\alpha_{i}}{\alpha_{j}}+\frac{\beta_{j}}{\beta_{i}} \geq 2-\varepsilon .
$$

By Lemma 3.5, this implies $\left|\alpha_{i}-\beta_{i}\right|<\varepsilon$, for all $i$; therefore

$$
\left\|P_{n}-Q_{n}\right\|=\max _{1 \leq i \leq p}\left|\alpha_{i}-\beta_{i}\right|<\varepsilon,
$$

so we obtain $P=Q$.

(ii) implies (i) is immediate from Lemma 2.1.

Corollary 3.7. Let the pair $(P, Q)$ of operators satisfy the condition of Theorem 3.6. Then the following properties are equivalent:

(i) $\forall X \in L(H),\left\|P X P^{-1}+Q^{-1} X Q\right\| \geq 2\|X\|$.

(ii) $P=Q$.

\section{Characterization of the Corach-Porta-Recht Inequality}

Notation. For $\theta \in\left[0, \pi\left[\right.\right.$, we denote by $D_{\theta}$ the straight line through the origin in the complex plane.

Lemma 4.1. Let $\lambda, \mu \in \mathbb{C}^{*}$ such that $\frac{\lambda}{\mu}+\frac{\mu}{\lambda} \in \mathbb{R}$ and $\left|\frac{\lambda}{\mu}+\frac{\mu}{\lambda}\right| \geq 2$. Then there exists a scalar $\theta \in\left[0, \pi\left[\right.\right.$ such that $\lambda, \mu \in D_{\theta}$. 
Proof. Let $\lambda=r_{1} e^{i \theta_{1}}$ and $\mu=r_{2} e^{i \theta_{2}}$ be the polar decompositions respectively of $\lambda$ and $\mu$. Then we have

$$
\frac{\lambda}{\mu}+\frac{\mu}{\lambda}=\left(\frac{r_{1}}{r_{2}}+\frac{r_{2}}{r_{1}}\right) \cos \left(\theta_{1}-\theta_{2}\right)+i\left(\frac{r_{1}}{r_{2}}-\frac{r_{2}}{r_{1}}\right) \sin \left(\theta_{1}-\theta_{2}\right),
$$

so we obtain $r_{1}=r_{2}$ or $\theta_{1}-\theta_{2} \equiv 0(\bmod . \pi)$.

The case $r_{1}=r_{2}$ also gives $\theta_{1}-\theta_{2} \equiv 0(\bmod . \pi)$, therefore $\lambda, \mu \in D_{\theta}$, for some $\theta \in[0, \pi[$.

Lemma 4.2. All invertible operators $S$ satisfying the condition

$$
\forall X \in L(H),\left\|S X S^{-1}+S^{-1} X S\right\| \geq 2\|X\|
$$

are normal.

Let $S$ be an invertible operator satisfying (5) and let $U P, V Q$ be the polar decompositions respectively of $S$ and $S^{*}$. Then, by (5), we obtain

$$
\forall X \in L(H),\left\|P X P^{-1}\right\|+\left\|Q^{-1} X Q\right\| \geq 2\|X\| .
$$

Since $P^{2}=S^{*} S$ and $Q^{2}=S S^{*}$, then $\sigma\left(P^{2}\right)=\sigma\left(Q^{2}\right)$, and by the spectral theorem, we obtain $\sigma(P)=\sigma(Q)$; so we have, by Theorem 3.6, $P=Q$, and also $S^{*} S=S S^{*}$. Therefore $S$ is normal.

Lemma 4.3. Let $S$ be an invertible normal operator. Then the following properties are equivalent:

(i) $\forall X \in L(H),\left\|S X S^{-1}+S^{-1} X S\right\| \geq 2\|X\|$.

(ii) $\sigma(S) \subset D_{\theta}$, for some $\theta \in[0, \pi[$.

(iii) $S=\lambda M$, for some nonzero scalar $\lambda$ and for some invertible self-adjoint operator $M$.

Proof. (i) implies (ii).

From (i) and Lemma 4.2, $S$ is normal. Then, by the spectral measure of $S$, there exists a sequence $\left(S_{n}\right)$ of invertible normal operators with finite spectrum such that

(a) $S_{n} \longrightarrow S$ uniformly,

(b) for all $\lambda$ in $\sigma(S)$, there exists a sequence $\left(\lambda_{n}\right)$ such that $\lambda_{n} \in \sigma\left(S_{n}\right)$, for all $n$ and $\lambda_{n} \longrightarrow \lambda$.

Let $\lambda, \mu \in \sigma(S)$ and let $\varepsilon>0$. Then by (i), (a) and (b), there exists an integer $N$ such that

$$
\forall n>N, \forall X \in L(H) \quad\left\|S_{n} X S_{n}^{-1}+S_{n}^{-1} X S_{n}\right\| \geq(2-\varepsilon)\|X\|
$$

and there exist two sequences $\left(\lambda_{n}\right)$ and $\left(\mu_{n}\right)$ such that

$$
\forall n, \lambda_{n}, \mu_{n} \in \sigma\left(S_{n}\right) ; \lambda_{n} \longrightarrow \lambda, \mu_{n} \longrightarrow \mu .
$$

Let $n>N$ and since $S_{n}$ is normal with finite spectrum, there exist $p$ orthogonal projections $E_{1}, \ldots, E_{p}$ such that $E_{k} E_{j}=E_{j} E_{k}=0$, if $k \neq j, E_{1} \oplus \ldots \oplus E_{p}=I$ and $S_{n}=\sum_{k=1}^{p} \alpha_{k} E_{k}$, where $\sigma\left(S_{n}\right)=\left\{\alpha_{1}, \ldots, \alpha_{p}\right\}, \alpha_{1}=\lambda_{n}, \alpha_{2}=\mu_{n}$.

Then by (6) and if we put $A=\left[\begin{array}{cc}2 & \gamma_{n} \\ \gamma_{n} & 2\end{array}\right]$, where $\gamma_{n}=\frac{\lambda_{n}}{\mu_{n}}+\frac{\mu_{n}}{\lambda_{n}}$, we obtain

$$
\forall X \in L\left(\mathbb{C}^{2}\right),\|A \circ X\| \geq(2-\varepsilon)\|X\|,
$$


and if we put $\delta_{n}=\frac{1}{\gamma_{n}}$ and $B=\left[\begin{array}{cc}\frac{1}{2} & \delta_{n} \\ \delta_{n} & \frac{1}{2}\end{array}\right]$, then from (7), we also have

$$
\forall X \in L\left(\mathbb{C}^{2}\right),\|B \circ X\| \leq \frac{\|X\|}{(2-\varepsilon)} .
$$

From (7), we deduce $\left|\frac{\lambda_{n}}{\mu_{n}}+\frac{\mu_{n}}{\lambda_{n}}\right| \geq(2-\varepsilon)$, so we obtain $\left|\frac{\lambda}{\mu}+\frac{\mu}{\lambda}\right| \geq 2$.

On the other hand, if in (8) we put $X=\left[\begin{array}{cc}1 & i a \\ i a & 1\end{array}\right]$, where $a>0$, we obtain $\frac{1}{4}+$ $a^{2}\left|\gamma_{n}\right|^{2}+a\left|\beta_{n}\right| \leq \frac{1+a^{2}}{(2-\varepsilon)^{2}}$, where $\beta_{n}=\operatorname{Im} \gamma_{n}$; so that $\frac{1}{4}+a^{2}|\alpha|^{2}+a|\beta| \leq \frac{1+a^{2}}{(2-\varepsilon)^{2}}$. Therefore $a|\alpha|^{2}+|\beta| \leq \frac{a}{4}$; then $\beta=0$ and $\frac{\lambda}{\mu}+\frac{\mu}{\lambda} \in \mathbb{R}$. This implies condition (ii) by Lemma 4.1 .

(ii) implies (iii).

If we put $M=e^{-i \theta} S$, then $M$ is an invertible normal operator with real spectrum, so we have $S=e^{i \theta} M$, where $M$ is an invertible self-adjoint operator.

(iii) implies (i) is immediate by Lemma 2.1.

Theorem 4.4. The set of all invertible operators $S$, for which

$$
\forall X \in L(H), \quad\left\|S X S^{-1}+S^{-1} X S\right\| \geq 2\|X\|
$$

is the set $\left\{\lambda M: \lambda \in \mathbb{C}^{*}, M\right.$ an invertible self-adjoint operator $\}$.

Proof. This follows immediately by Lemma 4.2 and Lemma 4.3.

Remark 4.1. The extremal class of invertible operators $S$ satisfying the condition

$$
\inf _{\|X\|=1}\left\|S X S^{-1}+S^{-1} X S\right\|=2
$$

has been characterized. So it remains the characterization of the second extremal class of all invertible operators $S$ satisfies the condition

$$
\inf _{\|X\|=1}\left\|S X S^{-1}+S^{-1} X S\right\|=0 .
$$

\section{REFERENCES}

[1] G. Corach, H. Porta, and L. Recht, An operator inequality. Linear Algebra Appl. 142 (1990), 153-158. MR 91m:47020

[2] J. P. Williams, Finite operators. Proc. Amer. Math. Soc. 26 (1970), 129-136. MR 41:9039

Department of Mathematics, Faculty of Science, University of Batna, 05000 Batna, Algeria

E-mail address: seddikameur@hotmail.com

Current address: Department of Mathematics, Faculty of Science, University of Sana'a, P.O. Box 14026, Sana'a, Yemen 